\title{
A Resilience Parameter Model Generated by a Compound Distribution
}

\author{
Hamid Bidram \\ Department of Statistics, University of Isfahan \\ Isfahan, 81746-73441, Iran \\ E-mail: h.bidram@sci.ui.ac.ir
}

Received: 19 July 2013; revised: 15 November 2013; accepted: 18 November 2013; published online: 9 December 2013

\begin{abstract}
In this paper, we shall attempt to extend the generalized exponential geometric distribution of Silva et al. [1]. The new four-parameter distribution also generalizes the Weibull-geometric distribution of Barreto-Souza et al. [2], exponentiated Weibull, and several other lifetime distributions as special cases. A useful characteristic of the new distribution is that its failure rate function can have different shapes. We first study certain basic distributional properties of the new distribution and provide closed form expressions for its moment generating function and moments. General expressions are also obtained for the order statistics densities and stress-strength parameter. Our findings happen to enfold several known results as special cases. The model parameters are estimated by the maximum likelihood method and the Fisher information matrix is discussed. Finally, the model is applied to a real data set and its advantage over some rival models is illustrated.

Key words: Beta Weibull, Beta Weibull-geometric, Maximum likelihood estimation, Order statistics, Resilience parameter family, Stress-strength parameter
\end{abstract}

\section{INTRODUCTION}

For many years, authors have been interested in developing methods for generating distributions with more flexibility for applications to data modeling. One of the remarkable methods, which has received much attention in the literature, is elevating the cumulative distribution function (cdf) of a random variable, say $F$, with a power parameter, say $\gamma>0$. The parameter $\gamma>0$ is called a resilience parameter and $\left\{F(. \mid \gamma)=F^{\gamma}(),. \gamma>0\right\}$ is a resilience parameter family or, alternatively, a proportional reverse hazards family, with underlying edf $F$ (see [3]). Clearly, $F(. \mid 1)=F($.).

In recent years, several distributions belonging to the resilience parameter family have been proposed in the literature. The exponentiated Weibull (EW) distribution which is a generalization of the Weibull distribution has been proposed by Mudholkar et al. [4], [5], and [6]. The generalized exponential (GE) distribution proposed by Gupta and Kundu [7], which extends the exponential distribution, is another remarkable distribution in the resilience parameter family. Nadarajah and
Kotz [8] presented four exponentiated type distributions that extend the standard gamma, standard Weibull, standard Gumbel, and standard Fréchet distributions. In addition, as some recent works, we can address the generalized exponentialPoisson (GEP) distribution proposed by Barreto-Souza and Cribari-Neto [9], the generalized modified Weibull (GMW) distribution proposed by Carrasco et al. [10], and the generalized exponential geometric (GEG) distribution given by Silva et al. [1] which extend the exponential-Poisson distribution of Kus [11], the modified Weibull distribution of Lai et al. [12], and the exponential-geometric (EG) distribution of Adamidis and Loukas [13], respectively. An alternative version of the GEG distribution is also given in [14].

In this paper, we attempt to extend the GEG distribution by raising the cdf of the Weibull-geometric (WG) distribution [2] to the power of a resilience parameter, say $\gamma>0$, whereas, the GEG distribution is obtained by elevating the cdf of an EG distribution to the power of $\gamma>0$. (For a comparison, see also Equations (1) and (13)). The main motivations for providing this paper include generalizing the GEG dis- 
tribution, providing failure rate function shapes, two general forms for order statistics densities and stress-strength parameter for distributions generated by the resilience parameter family, and also illustrating the advantage of the new distribution over some competitive models by applying it to a real data set.

In Section 2, the new distribution is proposed and its basic distributional properties such as the cumulative distribution, density, survival, failure rate, and moment generating functions as well as the moments and order statistics distributions are studied. The maximum likelihood method and Fisher information matrix are discussed in Section 3. Estimation of the stress-strength parameter with a general form is given in Section 4. Section 5 is devoted to the capacity of the new distribution in data modeling using a real data set. Some concluding remarks are given in Section 6.

\section{PROPOSED DISTRIBUTION AND ITS BASIC DISTRIBUTIONAL PROPERTIES}

Barreto-Souza et al. [2] proposed the WG distribution with the cdf

$$
G(x ; p, \beta, \alpha)=\frac{1-e^{-(\beta x)^{\alpha}}}{1-p e^{-(\beta x)^{\alpha}}} ; \quad x>0,
$$

where $p \in(0,1), \beta>0$, and $\alpha>0$ are constants. Now, we consider the following new cdf

$$
F(x ; p, \beta, \alpha, \gamma)=\left(\frac{1-e^{-(\beta x)^{\alpha}}}{1-p e^{-(\beta x)^{\alpha}}}\right)^{\gamma} ; \quad x>0,
$$

where $\gamma>0$ is a resilience parameter. A random variable as $X$ with the cdf given in (1) is said to have the extended generalized exponential geometric (EGEG) (or exponentiated Weibull-geometric) distribution with the notation $\operatorname{EGEG}(p, \beta, \alpha, \gamma)$. As two characteristics of the $\operatorname{EGEG}(p, \beta, \alpha, \gamma)$ distribution, when $\gamma>0$ is an integer, we may note that the cdf of $\max \left(Y_{1}, Y_{2}, \ldots, Y_{\gamma}\right)$ coincides with the cdf given in (1), where $Y_{i}$ 's are $\gamma$ independent and identically distributed (iid) WG random variables. In addition, in the case of integer $\gamma>0, \operatorname{cdf}(1)$ can be considered as the cdf of the $\gamma$ th order statistic of $N$ iid Weibull random variables, where $N$ is a geometric random variable with the success probability $1-p$, denoted by $N \sim G e(1-p)$, and is independent of $X_{i}$ 's. That is, we have the following observations:

Lemma II.1 Let $X_{1}, X_{2}, \ldots X_{N}$ be iid random variables having an absolutely continuous distribution function $F$ with a density function $f$, where $N$ has a geometric distribution with probability mass function $P(N=n)=(1-p) p^{n-1}$; $n=1,2, \ldots$, denoted by $N \sim G e(1-p)$, and is independent of $X_{i}$ 's. Then, the density, cumulative distribution, and survival functions of $U=\min \left(X_{1}, X_{2}, \ldots X_{N}\right)$ are, respectively, given by

$$
\begin{aligned}
f_{U}(x) & =\frac{(1-p) f(x)}{(1-p \bar{F}(x))^{2}}, \\
F_{U}(x) & =\frac{F(x)}{1-p \bar{F}(x)}
\end{aligned}
$$

and

$$
\bar{F}_{U}(x)=\frac{(1-p) \bar{F}(x)}{1-p \bar{F}(x)},
$$

for all $x \in \mathbb{R}=(-\infty,+\infty)$, where $\bar{F}=1-F$.

Proof. Using a conditional argument, the assertion follows.

Since the distribution of $U$ belongs to the Marshall and Olkin family [15], Lemma II.1 can also be followed from [15].

Theorem II.1 Under conditions given in Lemma II.1, the density and cumulative distribution functions of the rth order statistic of a random sample $X_{1}, X_{2}, \ldots X_{N}$ are given by

$$
f_{r}(x)=\frac{r(1-p) f(x) F(x)^{r-1}}{\{1-p \bar{F}(x)\}^{r+1}} ; \quad x \in \mathbb{R}
$$

and

$$
F_{r}(x)=\left[F_{U}(x)\right]^{r} ; \quad x \in \mathbb{R},
$$

respectively, where $F_{U}(x)$ is as given in (2).

Proof. The density function of the $r$ th order statistic, when $N$ has a general discrete distribution $\pi_{N}(n) ; n=1,2, \ldots$, is given by

$f_{r}(x)=\frac{1}{(r-1) ! \pi_{r}} f(x) F(x)^{r-1} G_{N}^{(r)}(\bar{F}(x)) ; \quad x \in \mathbb{R}$,

where $\pi_{r}=P(N \geq r)=\sum_{n=r}^{\infty} \pi_{N}(n)$ and $G_{N}($.$) denotes$ the probability generating function (pgf) of $N$ defined by

$$
G_{N}(z)=E\left(Z^{N}\right)=\sum_{n=1}^{\infty} z^{n} \pi_{N}(n)
$$

and $G_{N}^{(r)}(z)$ is its $r$ th derivative (see [16]). Since here $N$ has a geometric distribution, (6) reduces to

$$
G_{N}(z)=\frac{(1-p) z}{1-p z} ; \quad|z|<1
$$

and, thus, its $r$ th derivative is given by

$$
G_{N}^{(r)}(z)=\frac{(1-p) r ! p^{r-1}}{(1-p z)^{r+1}} ; \quad r=1,2, \ldots .
$$

In addition, in this case,

$$
\pi_{r}=\sum_{n=r}^{\infty}(1-p) p^{n-1}=p^{r-1}
$$


and, therefore, by inserting (7) and (8) into (5), the density function given in (3) is immediately achieved. Equation (4) is then obtained by integrating from (3) and the proof is completed.

As another example in this connection, we can address the GEG distribution of Silva et al. [1]. In fact, using another motivation different to that of Silva et al. [1], we can say that if $X_{1}, X_{2}, \ldots X_{N}$ is a random sample from an exponential distribution, where $N \sim G e(1-p)$ is independent of $X_{i}$ 's, then the distribution of the $r$ th order statistic of $X_{i}$ 's agrees with the GEG distribution, when its resilience parameter is an integer (see the cdf and density function of the GEG distribution given in (13) and (14), respectively).

\section{1. Density, survival, and failure rate functions}

Let $X$ be a random variable following the $\operatorname{EGEG}(p, \beta, \alpha, \gamma)$ distribution. Clearly, the corresponding probability density function (pdf) associated with the cdf (1) is given by

$$
\begin{aligned}
& f(x ; p, \beta, \alpha, \gamma)= \\
= & \frac{\gamma \alpha \beta^{\alpha}(1-p) x^{\alpha-1} e^{-(\beta x)^{\alpha}}\left(1-e^{-(\beta x)^{\alpha}}\right)^{\gamma-1}}{\left(1-p e^{-(\beta x)^{\alpha}}\right)^{\gamma+1}} ; x>0,
\end{aligned}
$$

where $\alpha>0$ and $\gamma>0$ are shape parameters, $\beta>0$ is a scale parameter, and parameter $p \in(0,1)$ is called a tilt parameter (see [3]).

Theorem II.2 The density function of an $\operatorname{EGEG}(p, \beta, \alpha, \gamma)$ distribution is logconcave if $\alpha \geq 1, \gamma>1$, and $p \leq \frac{\gamma-1}{\gamma+1}$ and, thus, it is strongly unimodal. It is logconvex if $0<\alpha \leq 1$ and $0<\gamma \leq 1$ and, thus, it is unimodal with mode at the origin.

Proof. Define $\eta(x)=-f^{\prime}(x) / f(x)=-(\log f(x))^{\prime}$, where $f^{\prime}$ is the first derivative of $f$. Therefore,

$$
\begin{aligned}
& \eta(x)=-\frac{1}{x}\left\{\alpha-1-\alpha(\beta x)^{\alpha}\right. \\
& \left.+\frac{(\gamma-1)(\beta x)^{\alpha} \alpha e^{-(\beta x)^{\alpha}}}{1-e^{-(\beta x)^{\alpha}}}-\frac{(\gamma+1) p(\beta x)^{\alpha} \alpha e^{-(\beta x)^{\alpha}}}{1-p e^{-(\beta x)^{\alpha}}}\right\},
\end{aligned}
$$

whose first derivative is given by

$$
\begin{gathered}
\eta^{\prime}(x)=\frac{\alpha-1}{x^{2}}+\frac{\alpha(\beta x)^{\alpha}}{x^{2}}\{\alpha-1 \\
-\frac{(\gamma-1) e^{-(\beta x)^{\alpha}}\left[(\alpha-1)\left(1-e^{-(\beta x)^{\alpha}}\right)-\alpha(\beta x)^{\alpha}\right]}{\left(1-e^{\left.-(\beta x)^{\alpha}\right)^{2}}\right.} \\
\left.+\frac{(\gamma+1) p e^{-(\beta x)^{\alpha}}\left[(\alpha-1)\left(1-p e^{-(\beta x)^{\alpha}}\right)-\alpha(\beta x)^{\alpha}\right]}{\left(1-p e^{-(\beta x)^{\alpha}}\right)^{2}}\right\} .
\end{gathered}
$$

It can be shown that

$$
\begin{aligned}
& \quad \frac{(\gamma+1) p\left[(\alpha-1)\left(1-p e^{-(\beta x)^{\alpha}}\right)-\alpha(\beta x)^{\alpha}\right]}{\left(1-p e^{-(\beta x)^{\alpha}}\right)^{2}}> \\
& >\frac{(\gamma-1)\left[(\alpha-1)\left(1-e^{-(\beta x)^{\alpha}}\right)-\alpha(\beta x)^{\alpha}\right]}{\left(1-e^{-(\beta x)^{\alpha}}\right)^{2}},
\end{aligned}
$$

for all $x>0, \beta>0, p \in(0,1), \alpha>0, \gamma>1$, and $p \leq \frac{\gamma-1}{\gamma+1}$. A proof of (11) is provided in the appendix. Therefore, if $\alpha \geq 1$, then (10) implies that $\eta^{\prime}(x)>0$ and, thus, the logconcavity of $f$ is proved in this case. In addition, since both functions $(\alpha-1)\left(1-e^{-(\beta x)^{\alpha}}\right)-\alpha(\beta x)^{\alpha}$ and $(\alpha-1)\left(1-p e^{-(\beta x)^{\alpha}}\right)-\alpha(\beta x)^{\alpha}$ are negative for all $x>0$, $0<\alpha \leq 1, \beta>0$, and $p \in(0,1)$, (10) implies that $\eta^{\prime}(x)<0$, when $0<\gamma \leq 1$. Thus, the logconvexity of $f$ is immediately explored in this case. Since it is known that logconcave densities are strongly unimodal and logconvex densities are decreasing with mode at the infimum of their support, i.e., here the origin (see, e.g., [17]), the proof is completed.

Fig. 1 plots the density function of an $\operatorname{EGEG}(p, \beta, \alpha, \gamma)$ distribution for different parameter values. It is easily seen that the survival and failure rate functions of an $\operatorname{EGEG}(p, \beta, \alpha, \gamma)$ distribution are given by

$$
\bar{F}(x ; p, \beta, \alpha, \gamma)=1-\left(\frac{1-e^{-(\beta x)^{\alpha}}}{1-p e^{-(\beta x)^{\alpha}}}\right)^{\gamma} ; x>0,
$$

and

$$
\begin{aligned}
& h(x ; p, \beta, \alpha, \gamma)= \\
& =\frac{\gamma \alpha \beta^{\alpha}(1-p) x^{\alpha-1} e^{-(\beta x)^{\alpha}}\left(1-e^{-(\beta x)^{\alpha}}\right)^{\gamma-1}}{\left(1-p e^{-(\beta x)^{\alpha}}\right)\left\{\left(1-p e^{-(\beta x)^{\alpha}}\right)^{\gamma}\right.} ; x>0, \\
& \left.-\left(1-e^{-(\beta x)^{\alpha}}\right)^{\gamma}\right\}
\end{aligned}
$$

respectively.

Corollary II.1 The failure rate function of an $E G E G(p, \beta$, $\alpha, \gamma)$ distribution is increasing if $\alpha \geq 1, \gamma>1$, and $p \leq \frac{\gamma-1}{\gamma+1}$ and it is decreasing if $0<\alpha \leq 1$ and $0<\gamma \leq 1$.

Proof. According to the proof of Theorem II.2, we have $\eta^{\prime}(x)>0$ for $\alpha \geq 1, \gamma>1$, and $p \leq \frac{\gamma-1}{\gamma+1}$. Thus, Glaser's [18] Theorem implies that the failure rate is increasing in this case. In addition, we have $\eta^{\prime}(x)<0$, for $0<\alpha \leq 1$ and $0<\gamma \leq 1$. Therefore, Glaser's [18] Theorem again implies that the failure rate is decreasing in this case.

Fig. 2 plots the failure rate function of an $\operatorname{EGEG}(p, \beta, \alpha, \gamma)$ distribution for different selected parameter values which contains the failure rate shapes of some previously known distributions. As we see from Fig. 2, the failure rate function can also take bathtub and upside-down bathtub shapes for other values of the parameters.

As an important property of the resilience parameter family, we see that the reversed failure rate function of an 

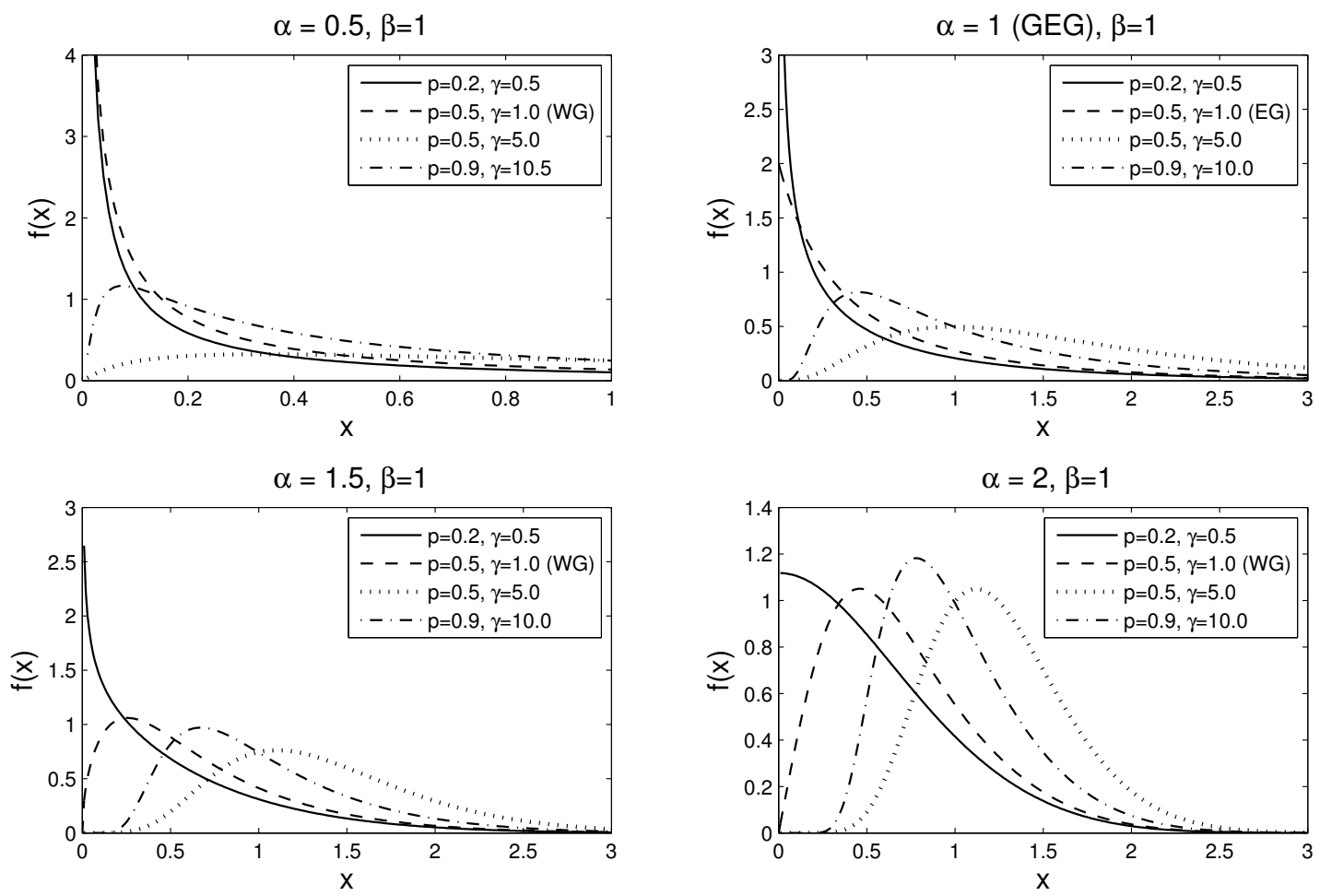

Fig. 1. Plots of an $E G E G(p, \beta, \alpha, \gamma)$ density function for different parameter values
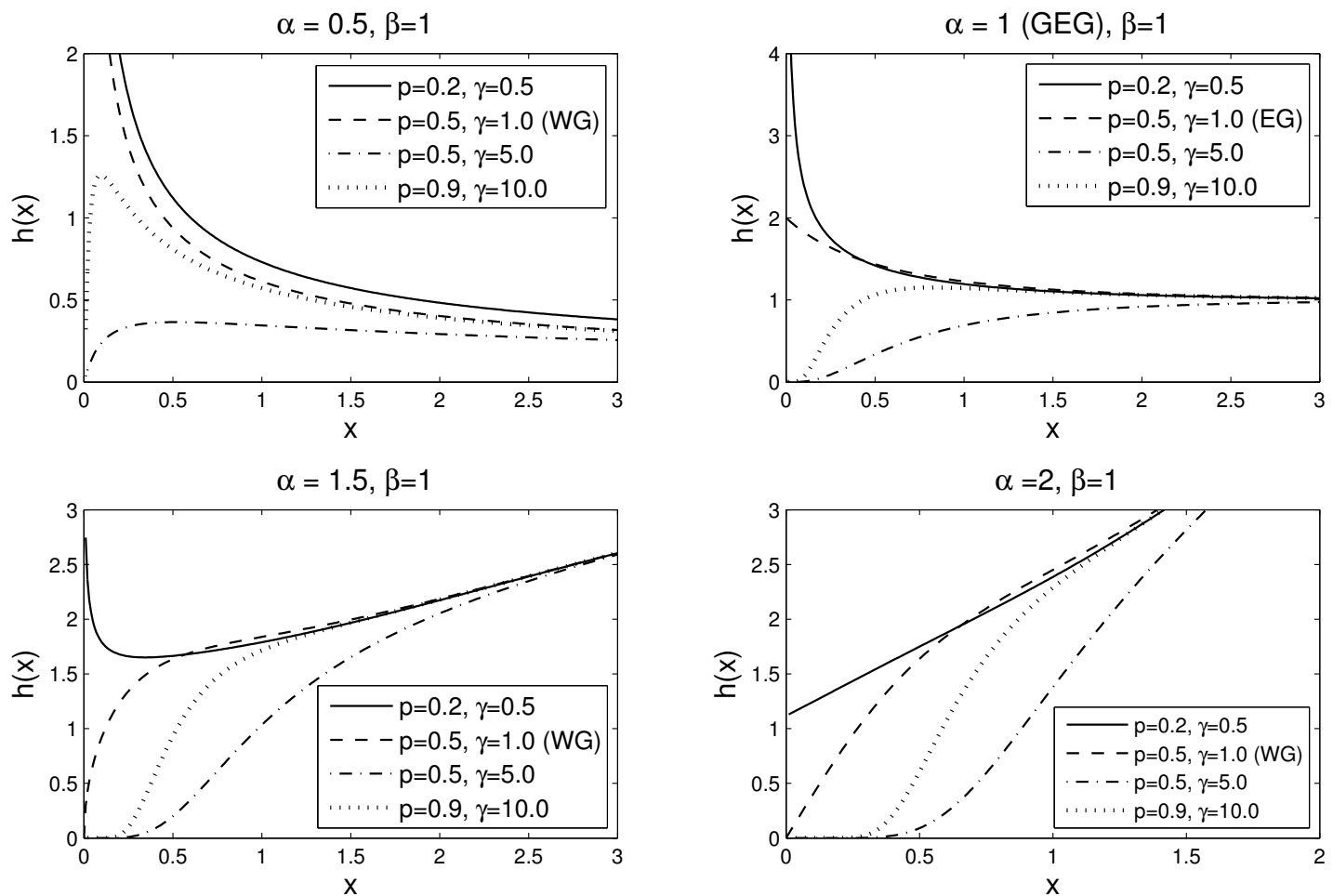

Fig. 2. Plots of an EGEG failure rate function for some selected parameter values 


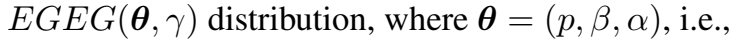

$$
r_{E G E G}(x ; \boldsymbol{\theta}, \gamma)=\frac{f_{E G E G}(x ; \boldsymbol{\theta}, \gamma)}{F_{E G E G}(x ; \boldsymbol{\theta}, \gamma)}=\gamma r_{W G}(x ; \boldsymbol{\theta}),
$$

is proportional to the reversed failure rate function of the $W G(\boldsymbol{\theta})$ distribution.

A simulation of the $\operatorname{EGE} G(p, \beta, \alpha, \gamma)$ random variable can be obtained by the relationship $X=\frac{1}{\beta}\left\{\log \frac{1-p U^{\frac{1}{\gamma}}}{1-U^{\frac{1}{\gamma}}}\right\}^{\frac{1}{\alpha}}$. The $q$ th quantile $x_{q}$ of an $E G E G(p, \beta, \alpha, \gamma)$ distribution is given by $x_{q}=\frac{1}{\beta}\left\{\log \frac{1-p q^{\frac{1}{\gamma}}}{1-q^{\frac{1}{\gamma}}}\right\}^{\frac{1}{\alpha}}$, and, consequently, its median equals $m=\frac{1}{\beta}\left\{\log \frac{1-p(0.5)^{\frac{1}{\gamma}}}{1-(0.5)^{\frac{1}{\gamma}}}\right\}^{\frac{1}{\alpha}}$.

\section{2. Some known special cases}

Below, we observe that the family of $\operatorname{EGEG}(p, \beta, \alpha, \gamma)$ distributions contains several previously known distributions as special cases.

1. When $\gamma=1$, the $E G E G(p, \beta, \alpha, \gamma)$ distribution reduces to the $W G(p, \beta, \alpha)$ distribution of Barreto-Souza et al. [2] with pdf

$$
\begin{aligned}
& f_{W G}(x ; p, \beta, \alpha)= \\
= & \alpha \beta^{\alpha}(1-p) x^{\alpha-1} e^{-(\beta x)^{\alpha}}\left(1-p e^{-(\beta x)^{\alpha}}\right)^{-2} ; \quad x>0 .
\end{aligned}
$$

2. When $\gamma=\alpha=1$, we obtain the $E G(p, \beta)$ distribution of Adamidis and Loukas [13] with pdf

$f_{E G}(x ; p, \beta)=\beta(1-p) e^{-\beta x}\left(1-p e^{-\beta x}\right)^{-2} ; \quad x>0$,

which reduces to an exponential distribution with parameter $\beta>0$, when $p \rightarrow 0^{+}$.

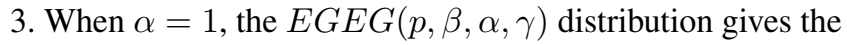
$\operatorname{GEG}(p, \beta, \gamma)$ distribution of Silva et al. [1] with the cdf and pdf

$$
F_{G E G}(x ; p, \beta, \gamma)=\left\{\frac{1-e^{-(\beta x)}}{1-p e^{-\beta x}}\right\}^{\gamma} ; x>0,
$$

and

$$
\begin{aligned}
f_{G E G}(x ; p, \beta, \gamma) & =\gamma \beta(1-p) e^{-\beta x}\left(1-e^{-\beta x}\right)^{\gamma-1} \\
& \times\left(1-p e^{-\beta x}\right)^{-(\gamma+1)} ; \quad x>0,
\end{aligned}
$$

respectively, which reduces to a GE distribution with parameters $\gamma>0$ and $\beta>0$, when $p \rightarrow 0^{+}$.

4. When $p \rightarrow 0^{+}$, the $\operatorname{EGEG}(p, \beta, \alpha, \gamma)$ distribution converges to the $E W(\beta, \alpha, \gamma)$ distribution (see, e.g., [4]) with pdf

$$
\begin{array}{r}
f_{E W}(x ; \beta, \alpha, \gamma)=\gamma \alpha \beta^{\alpha} x^{\alpha-1} e^{-(\beta x)^{\alpha}}\left(1-e^{-(\beta x)^{\alpha}}\right)^{\gamma-1} \\
x>0,
\end{array}
$$

which reduces to a Weibull distribution with parameters $\alpha>0$ and $\beta>0$, when $\gamma=1$.

5. The $E G E G(p, \beta, \alpha, \gamma)$ distribution reduces to a degenerated distribution at the origin, when $p \rightarrow 1^{-}$.

\section{3. Moment generating function and moments}

It seems that mgf of an $\operatorname{EGEG}(p, \beta, \alpha, \gamma)$ distribution is not easily found directly from the density function given in (9). Hence, we first obtain an alternative expression for the density function of the $E G E G(p, \beta, \alpha, \gamma)$ distribution using the series representation

$$
(1-z)^{-k}=\sum_{i=0}^{\infty} \frac{\Gamma(k+i)}{\Gamma(k) i !} z^{i} ; \quad|z|<1, k>0 .
$$

Then, we obtain a closed form for the mgf of the $E G E G(p, \beta, \alpha, \gamma)$ distribution and show that our result contains certain known results in this connection. Using (16), pdf (9) can be represented by

$$
\begin{aligned}
f(x)=\sum_{i=0}^{\infty}(1-p) p^{i} \frac{\alpha \beta^{\alpha}}{B(\gamma, i+1)} x^{\alpha-1} e^{-(i+1)(\beta x)^{\alpha}} \\
\quad \times\left(1-e^{-(\beta x)^{\alpha}}\right)^{\gamma-1}= \\
=(1-p) \sum_{i=0}^{\infty} p^{i} f_{B W}(x ; \gamma, i+1, \beta, \alpha)
\end{aligned}
$$

where

$$
\begin{aligned}
& f_{B W}(x ; a, b, \lambda, c)=\frac{c \lambda^{c}}{B(a, b)} x^{c-1} e^{-b(\lambda x)^{c}} \\
\times & \left\{1-e^{-(\lambda x)^{c}}\right\}^{a-1} ; x>0,
\end{aligned}
$$

is the density function of the beta Weibull (BW) distribution proposed by Cordeiro et al. [19] with parameters $a>0, b>$ $0, \lambda>0$, and $c>0$, where $B(a, b)=\int_{0}^{1} t^{a-1}(1-t)^{b-1} d t$ is the beta function. The beta Weibull distribution has also been studied by Famoye et al. [20] and Lee et al. [21]. As we see from (17), the $E G E G(p, \beta, \alpha, \gamma)$ density function is an infinite weighted sum of BW densities with a geometric weight. Hence, we can obtain some of its properties, such as the mgf and moments, directly from those of the BW distribution.

For a random variable $Y$ following the $B W(a, b, \lambda, c)$ distribution, Cordeiro et al. [19] showed that the $\operatorname{mgf}$ of $Y$, for real non-integer $a>0$, is given by

$$
M_{Y}(t)=\frac{\Gamma(a)}{B(a, b)} \sum_{r=0}^{\infty} \sum_{j=0}^{\infty} \frac{t^{r} \Gamma(r / c+1)(-1)^{j}}{\lambda^{r} \Gamma(a-j)(b+j)^{r / c+1} r ! j !},
$$


and, when $a>0$ is integer, it is given by

$$
\begin{aligned}
& M_{Y}(t)= \\
= & \frac{1}{B(a, b)} \sum_{r=0}^{\infty} \frac{t^{r} \Gamma(r / c+1)}{\lambda^{r} r !} \sum_{j=0}^{a-1}\left(\begin{array}{c}
a-1 \\
j
\end{array}\right) \frac{(-1)^{j}}{(b+j)^{r / c+1}} .
\end{aligned}
$$

Now, combining Eqs. (17) and (18), for real non-integer $\gamma>0$, yields

$$
\begin{aligned}
M_{X}(t) & =(1-p) \\
& \times \sum_{i=0}^{\infty} \sum_{r=0}^{\infty} \sum_{j=0}^{\infty} p^{i} \frac{\Gamma(\gamma+i+1) t^{r} \Gamma(r / \alpha+1)(-1)^{j}}{\beta^{r} \Gamma(\gamma-j)(i+1+j)^{r / \alpha+1} i ! r ! j !},
\end{aligned}
$$

and, when $\gamma>0$ is integer, by combining Eqs. (17) and (19), we have

$$
\begin{aligned}
& M_{X}(t)=(1-p) \\
\times & \sum_{i=0}^{\infty} \sum_{r=0}^{\infty} \sum_{j=0}^{\gamma-1} p^{i} \frac{(\gamma+i) ! t^{r} \Gamma(r / \alpha+1)(-1)^{j}}{\beta^{r}(\gamma-1-j) !(i+1+j)^{r / \alpha+1} i ! r ! j !} .
\end{aligned}
$$

In particular, for $\gamma=1$, Equation (20) reduces to

$$
M_{Z}(t)=(1-p) \sum_{i=0}^{\infty} \sum_{r=0}^{\infty} p^{i} \frac{t^{r} \Gamma(r / \alpha+1)}{\beta^{r} r !(i+1)^{r / \alpha}},
$$

where $Z$ is a WG random variable with parameters $p \in(0,1)$, $\beta>0$, and $\alpha>0$ (see also [22]).

Cordeiro et al. [19] showed that the $m$ th moment of the BW distribution, for real non-integer and integer values of $a>0$, respectively, are as given below:

$$
\begin{aligned}
E\left(Y^{m}\right) & =\frac{\Gamma(a) \Gamma(m / c+1)}{\lambda^{m} B(a, b)} \\
& \times \sum_{j=0}^{\infty} \frac{(-1)^{j}}{\Gamma(a-j) j !(b+j)^{m / c+1}} ; \quad m=1,2, \ldots,
\end{aligned}
$$

and

$$
\begin{array}{r}
E\left(Y^{m}\right)=\frac{\Gamma(m / c+1)}{\lambda^{m} B(a, b)} \sum_{j=0}^{a-1}\left(\begin{array}{c}
a-1 \\
j
\end{array}\right) \frac{(-1)^{j}}{(b+j)^{m / c+1}} \\
m=1,2, \ldots
\end{array}
$$

Thus, by (17), it readily follows that the $m$ th moment of the $\operatorname{EGEG}(p, \beta, \alpha, \gamma)$ distribution, for a non-integer $\gamma>0$, is given by

$$
\begin{aligned}
& E\left(X^{m}\right)=\frac{(1-p) \Gamma(m / \alpha+1)}{\beta^{m}} \\
\times & \sum_{i=0}^{\infty} \sum_{j=0}^{\infty} \frac{p^{i} \Gamma(\gamma+i+1)(-1)^{j}}{\Gamma(\gamma-j)(i+1+j)^{m / \alpha+1} i ! j !} ; \quad m=1,2, \ldots,
\end{aligned}
$$

which reduces to

$$
\begin{aligned}
& E\left(X^{m}\right)=\frac{(1-p) \Gamma(m / \alpha+1)}{\beta^{m}} \\
\times & \sum_{i=0}^{\infty} \sum_{j=0}^{\gamma-1} \frac{p^{i}(\gamma+i) !(-1)^{j}}{(\gamma-j-1) !(i+1+j)^{m / \alpha+1} i ! j !} ; m=1,2, \ldots,
\end{aligned}
$$

when $\gamma>0$ is an integer value. In particular, when $\gamma=1$, Equation (22) becomes

$$
\begin{aligned}
E\left(Z^{m}\right) & =\frac{(1-p) \Gamma(m / \alpha+1)}{\beta^{m}} \sum_{i=0}^{\infty} p^{i}(i+1)^{-m / \alpha}= \\
& =\frac{(1-p) \Gamma(m / \alpha+1)}{p \beta^{m}} L(p, m / \alpha),
\end{aligned}
$$

where $L(p, a)=\sum_{j=1}^{\infty} p^{j} j^{-a}$ is Euler's polylogarithm function (Erdelyi et al. [23]) and $Z$ is as in (21).

\section{4. Order statistics}

Let $X_{1}, X_{2}, \ldots, X_{n}$ be a random sample from an $\operatorname{EGEG}(\boldsymbol{\theta}, \gamma)$ distribution, where $\boldsymbol{\theta}=(p, \beta, \alpha)$. Then, the density function of the $i$ th order statistic is given by

$$
\begin{aligned}
& f_{i: n}(x ; \boldsymbol{\theta}, \gamma)= \\
= & \frac{1}{B(i, n-i+1)} \\
\times & f(x ; \boldsymbol{\theta}, \gamma)[F(x ; \boldsymbol{\theta}, \gamma)]^{i-1}[1-F(x ; \boldsymbol{\theta}, \gamma)]^{n-i} \\
= & \frac{1}{B(i, n-i+1)} \\
\times & \sum_{k=0}^{n-i}\left(\begin{array}{c}
n-i \\
k
\end{array}\right)(-1)^{k} f(x ; \boldsymbol{\theta}, \gamma)[F(x ; \boldsymbol{\theta}, \gamma)]^{k+i-1} \\
= & \frac{1}{B(i, n-i+1)} \\
\times & \sum_{k=0}^{n-i}\left(\begin{array}{c}
n-i \\
k
\end{array}\right)(-1)^{k} g(x ; \boldsymbol{\theta})\left[G(x ; \boldsymbol{\theta}]^{\gamma(k+i)-1}\right. \\
= & \frac{1}{B(i, n-i+1)} \\
\times & \sum_{k=0}^{n-i} \frac{(-1)^{k}\left(\begin{array}{c}
n-i \\
k
\end{array}\right)}{i+k} f_{E G E G}(x ; \boldsymbol{\theta}, \gamma(i+k)),
\end{aligned}
$$

where $g(x ; \boldsymbol{\theta})$ and $G(x ; \boldsymbol{\theta})$ are the density function and cdf of the $W G(\boldsymbol{\theta})$ distribution, respectively. Note that (23) is a general form, i.e., it holds for any distribution belonging to the resilience parameter family. For example, the corresponding expression given by Silva et al. [1] for the $G E G(p, \beta, \gamma)$ distribution agrees with this general form. As we see, the density function of the $i$ th order statistic of an $\operatorname{EGEG}(p, \beta, \alpha, \gamma)$ distribution is a finite linear combination of $\operatorname{EGEG}(p, \beta, \alpha, \gamma(i+k))$ densities.Thus, 
we may obtain certain properties of the $i$ th order statistic of the $E G E G(p, \beta, \alpha, \gamma)$ distribution, such as its moments, directly from the corresponding properties of the $E G E G(p, \beta, \alpha, \gamma(i+k))$ distribution.

\section{ESTIMATION AND FISHER INFORMATION MATRIX}

Let $x_{1}, x_{2}, \ldots, x_{n}$ be $n$ observations from a random sample drawn from the EGEG distribution and $\boldsymbol{\theta}=(p, \beta, \alpha, \gamma)^{T}$ be the parameter vector. The log-likelihood function is given by

$$
\begin{aligned}
\ell & =n \log \left[\gamma \alpha \beta^{\alpha}(1-p)\right]+(\alpha-1) \sum_{i=1}^{n} \log x_{i} \\
& -\sum_{i=1}^{n}\left(\beta x_{i}\right)^{\alpha}+(\gamma-1) \sum_{i=1}^{n} \log \left(1-e^{-\left(\beta x_{i}\right)^{\alpha}}\right) \\
& -(\gamma+1) \sum_{i=1}^{n} \log \left(1-p e^{-\left(\beta x_{i}\right)^{\alpha}}\right) .
\end{aligned}
$$

The components of the score vector $U=\left(\frac{\partial \ell}{\partial p}, \frac{\partial \ell}{\partial \beta}, \frac{\partial \ell}{\partial \alpha}, \frac{\partial \ell}{\partial \gamma}\right)^{T}$ are given by

$$
\begin{aligned}
\frac{\partial \ell}{\partial p}= & \frac{-n}{1-p}+(\gamma+1) \sum_{i=1}^{n} \frac{e^{-\left(\beta x_{i}\right)^{\alpha}}}{1-p e^{-\left(\beta x_{i}\right)^{\alpha}}} \\
\frac{\partial \ell}{\partial \beta}= & \frac{n \alpha}{\beta}-\frac{\alpha}{\beta} \sum_{i=1}^{n}\left(\beta x_{i}\right)^{\alpha} \\
& +\frac{(\gamma-1) \alpha}{\beta} \sum_{i=1}^{n} \frac{\left(\beta x_{i}\right)^{\alpha} e^{-\left(\beta x_{i}\right)^{\alpha}}}{1-e^{-\left(\beta x_{i}\right)^{\alpha}}} \\
& -\frac{(\gamma+1) p \alpha}{\beta} \sum_{i=1}^{n} \frac{\left(\beta x_{i}\right)^{\alpha} e^{-\left(\beta x_{i}\right)^{\alpha}}}{1-p e^{-\left(\beta x_{i}\right)^{\alpha}}} \\
\frac{\partial \ell}{\partial \alpha}= & \frac{n}{\alpha}+n \log \beta+\sum_{i=1}^{n} \log x_{i} \\
& -\sum_{i=1}^{n}\left(\beta x_{i}\right)^{\alpha} \log \left(\beta x_{i}\right) \\
& +(\gamma-1) \sum_{i=1}^{n} \frac{\left(\beta x_{i}\right)^{\alpha} e^{-\left(\beta x_{i}\right)^{\alpha}} \log \left(\beta x_{i}\right)}{1-e^{-\left(\beta x_{i}\right)^{\alpha}}} \\
& -(\gamma+1) p \sum_{i=1}^{n} \frac{\left(\beta x_{i}\right)^{\alpha} e^{-\left(\beta x_{i}\right)^{\alpha}} \log \left(\beta x_{i}\right)}{1-p e^{-\left(\beta x_{i}\right)^{\alpha}}}
\end{aligned}
$$

and

$$
\frac{\partial \ell}{\partial \gamma}=\frac{n}{\gamma}+\sum_{i=1}^{n} \log \frac{1-e^{-\left(\beta x_{i}\right)^{\alpha}}}{1-p e^{-\left(\beta x_{i}\right)^{\alpha}}} .
$$

Using a numerical method such as the Newton-Raphson procedure, the maximum likelihood estimates (MLEs) of the parameters are obtained by solving the nonlinear equations $U(\hat{\boldsymbol{\theta}})=\mathbf{0}$. The total Fisher information matrix is given by $\boldsymbol{I}_{n}(\boldsymbol{\theta})=n \boldsymbol{I}(\boldsymbol{\theta})$, where

$$
\boldsymbol{I}(\boldsymbol{\theta})=\left[\begin{array}{cccc}
I_{p p} & I_{p \beta} & I_{p \alpha} & I_{p \gamma} \\
I_{\beta p} & I_{\beta \beta} & I_{\beta \alpha} & I_{\beta \gamma} \\
I_{\alpha p} & I_{\alpha \beta} & I_{\alpha \alpha} & I_{\alpha \gamma} \\
I_{\gamma p} & I_{\gamma \beta} & I_{\gamma \alpha} & I_{\gamma \gamma}
\end{array}\right]
$$

whose components can be calculated by

$$
I_{\theta_{i}, \theta_{j}}=-E\left(\frac{\partial^{2} \ell}{\partial \theta_{i} \theta_{j}}\right) ; i, j=1,2,3,4 .
$$

The above expectations can be obtained numerically by mathematical softwares such as MATLAB or MAPLE. However, the total Fisher information matrix can be approximated by

$$
\boldsymbol{I}_{n}(\hat{\boldsymbol{\theta}}) \approx-\left[\begin{array}{cccc}
\left.\frac{\partial^{2} \ell}{\partial p^{2}}\right|_{\hat{\boldsymbol{\theta}}} & \left.\frac{\partial^{2} \ell}{\partial p \partial}\right|_{\hat{\boldsymbol{\theta}}} & \left.\frac{\partial^{2} \ell}{\partial p \partial \alpha}\right|_{\hat{\boldsymbol{\theta}}} & \left.\frac{\partial^{2} \ell}{\partial p \partial \gamma}\right|_{\hat{\boldsymbol{\theta}}} \\
\left.\frac{\partial^{2} \ell}{\partial \partial \partial \partial}\right|_{\hat{\boldsymbol{\theta}}} & \left.\frac{\partial^{2} \ell}{\partial \beta^{2}}\right|_{\hat{\boldsymbol{\theta}}} & \left.\frac{\partial^{2} \ell}{\partial \beta \partial \alpha}\right|_{\hat{\boldsymbol{\theta}}} & \left.\frac{\partial^{2} \ell}{\partial \beta \partial \gamma}\right|_{\hat{\boldsymbol{\theta}}} \\
\left.\frac{\partial^{2} \ell}{\partial \alpha \partial p}\right|_{\hat{\boldsymbol{\theta}}} & \left.\frac{\partial^{2} \ell}{\partial \alpha \beta}\right|_{\hat{\boldsymbol{\theta}}} & \left.\frac{\partial^{2} \ell}{\partial \alpha^{2}}\right|_{\hat{\boldsymbol{\theta}}} & \left.\frac{\partial^{2} \ell}{\partial \alpha \partial \gamma}\right|_{\hat{\boldsymbol{\theta}}} \\
\left.\frac{\partial^{2} \ell}{\partial \gamma \partial p}\right|_{\hat{\boldsymbol{\theta}}} & \left.\frac{\partial^{2} \ell}{\partial \gamma \partial \beta}\right|_{\hat{\boldsymbol{\theta}}} & \left.\frac{\partial^{2} \ell}{\partial \gamma \partial \alpha}\right|_{\hat{\boldsymbol{\theta}}} & \left.\frac{\partial^{2} \ell}{\partial \gamma^{2}}\right|_{\hat{\boldsymbol{\theta}}}
\end{array}\right],
$$

where $\hat{\boldsymbol{\theta}}$ is MLE of $\boldsymbol{\theta}$. Under conditions given in Ferguson ( [24], pp:121), $\hat{\boldsymbol{\theta}}$ has an asymptotic distribution as $N_{4}\left(\boldsymbol{\theta}, \boldsymbol{I}_{n}(\hat{\boldsymbol{\theta}})^{-1}\right)$, or equivalently, $\boldsymbol{I}_{n}(\hat{\boldsymbol{\theta}})^{1 / 2}(\hat{\boldsymbol{\theta}}-\boldsymbol{\theta})^{T}$ has a multivariate standard normal distribution. The asymptotic normal distributions are usually used for constructing approximate confidence intervals, confidence regions, and testing of hypotheses of the parameters. For example, an asymptotic confidence interval with confidence coefficient $1-\gamma$ for parameters $\theta_{j} ; j=1,2,3,4$, is computed by $\left(\hat{\theta_{j}} \mp z_{1-\gamma / 2} \sqrt{I_{\hat{\theta_{j}}, \hat{\theta_{j}}}}\right)$ where $I_{\hat{\theta_{j}}, \hat{\theta_{j}}}$ is the $j$ th diagonal element of $\boldsymbol{I}_{n}(\hat{\boldsymbol{\theta}})^{-1}$ and $z_{1-\gamma / 2}$ is the $(1-\gamma / 2)$ th quantile of the standard normal distribution (see Section 5).

\section{ESTIMATION OF THE STRESS-STRENGTH PARAMETER}

In the context of reliability, the stress-strength parameter $R=P(X>Y)$ is a measure of component reliability and its estimation problem, when $X$ and $Y$ are independent and follow a specified distribution, has been discussed widely in the literature. Suppose that the random variable $X$ is the strength of a component which is subjected to a random stress $Y$. The component fails whenever $X<Y$ and there is no failure when $X>Y$. In this section, the $R$ parameter is estimated for the $E G E G(\boldsymbol{\theta}, \gamma)$ distribution, where $\boldsymbol{\theta}=(p, \beta, \alpha)$. We shall discuss the matter for different cases as below:

\section{1. The case $\gamma_{1} \neq \gamma_{2}$}

Let $X$ and $Y$ be independent random variables with

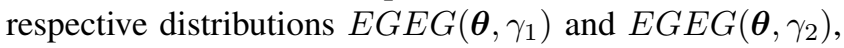


where $\boldsymbol{\theta}=(p, \beta, \alpha)$. The stress-strength parameter is given by

$$
\begin{aligned}
R & =P(X>Y)=\int_{0}^{\infty} f_{X}\left(x ; \boldsymbol{\theta}, \gamma_{1}\right) F_{Y}\left(x ; \boldsymbol{\theta}, \gamma_{2}\right) d x \\
& =\int_{0}^{\infty} \gamma_{1} g(x ; \boldsymbol{\theta}) G(x ; \boldsymbol{\theta})^{\gamma_{1}+\gamma_{2}} d x \\
& =\frac{\gamma_{1}}{\gamma_{1}+\gamma_{2}}
\end{aligned}
$$

where $g(x ; \boldsymbol{\theta})$ and $G(x ; \boldsymbol{\theta})$ are the density function and cdf of the $W G(\boldsymbol{\theta})$ distribution, respectively. As we see, (24) can be considered as a general form. For example, see the corresponding expression for the $G E G(p, \beta, \gamma)$ distribution given by Silva et al. [1].

Now, assume that $x_{1}, x_{2}, \ldots, x_{n}$ and $y_{1}, y_{2}, \ldots, y_{m}$ are independent observations of $X \sim \operatorname{EGEG}\left(p, \beta, \alpha, \gamma_{1}\right)$ and $Y \sim \operatorname{EGEG}\left(p, \beta, \alpha, \gamma_{2}\right)$, respectively. The total loglikelihood function $\ell_{R}\left(\boldsymbol{\theta}^{*}\right)$, where $\boldsymbol{\theta}^{*}=\left(p, \beta, \alpha, \gamma_{1}, \gamma_{2}\right)^{T}$, is given by

$$
\begin{aligned}
& \ell_{R}\left(\boldsymbol{\theta}^{*}\right)=n \log \gamma_{1}+m \log \gamma_{2} \\
& +(n+m) \log \left[\alpha \beta^{\alpha}(1-p)\right] \\
& +(\alpha-1)\left(\sum_{i=1}^{n} \log x_{i}+\sum_{j=1}^{m} \log y_{j}\right) \\
& -\sum_{i=1}^{n}\left(\beta x_{i}\right)^{\alpha}-\sum_{j=1}^{m}\left(\beta y_{j}\right)^{\alpha} \\
& +\left(\gamma_{1}-1\right) \sum_{i=1}^{n} \log \left(1-e^{-\left(\beta x_{i}\right)^{\alpha}}\right) \\
& +\left(\gamma_{2}-1\right) \sum_{j=1}^{m} \log \left(1-e^{-\left(\beta y_{j}\right)^{\alpha}}\right) \\
& -\left(\gamma_{1}+1\right) \sum_{i=1}^{n} \log \left(1-p e^{-\left(\beta x_{i}\right)^{\alpha}}\right) \\
& -\left(\gamma_{2}+1\right) \sum_{j=1}^{m} \log \left(1-p e^{-\left(\beta y_{j}\right)^{\alpha}}\right) .
\end{aligned}
$$

The MLE of $\boldsymbol{\theta}^{*}$, say $\hat{\boldsymbol{\theta}}^{*}$, can be calculated numerically from the system of nonlinear equation $U_{R}\left(\boldsymbol{\theta}^{*}\right)=$ $\left(\frac{\partial \ell_{R}}{\partial \hat{p}}, \frac{\partial \ell_{R}}{\partial \hat{\beta}}, \frac{\partial \ell_{R}}{\partial \hat{\alpha}}, \frac{\partial \ell_{R}}{\partial \hat{\gamma}_{1}}, \frac{\partial \ell_{R}}{\partial \hat{\gamma}_{2}}\right)^{T}=\mathbf{0}$. Hence, the parameter $R$ can be estimated by $\hat{R}=\frac{\hat{\gamma_{1}}}{\hat{\gamma_{1}}+\hat{\gamma_{2}}}$.

\section{2. The cases $\gamma_{1} \neq \gamma_{2}$ and $p_{1} \neq p_{2}$}

Assume that $X \sim \operatorname{EGEG}\left(p_{1}, \beta, \alpha, \gamma_{1}\right)$ and $Y \sim$ $\operatorname{EGEG}\left(p_{2}, \beta, \alpha, \gamma_{2}\right)$ are independent random variables.
In this case, the parameter $R$ can be written as

$$
\begin{aligned}
R & =\int_{0}^{\infty} \frac{\gamma_{1} \alpha \beta^{\alpha}\left(1-p_{1}\right) x^{\alpha-1} e^{-(\beta x)^{\alpha}}}{\left(1-p_{1} e^{-(\beta x)^{\alpha}}\right)^{\gamma_{1}+1}} \\
& \times\left(\frac{1-e^{-(\beta x)^{\alpha}}}{1-p_{2} e^{-(\beta x)^{\alpha}}}\right)^{\gamma_{2}} d x .
\end{aligned}
$$

Expanding the binomial terms $\left(1-p_{1} e^{-(\beta x)^{\alpha}}\right)^{\gamma_{1}+1}$ and $\left(1-p_{2} e^{-(\beta x)^{\alpha}}\right)^{\gamma_{2}}$, according to Equation (16), we obtain

$$
\begin{aligned}
R & =\frac{1-p_{1}}{B\left(\gamma_{1}, \gamma_{2}\right)\left(\gamma_{1}+\gamma_{2}\right)} \\
& \times \sum_{i=0}^{\infty} \sum_{j=0}^{\infty} p_{1}^{i} p_{2}^{j}\left(\begin{array}{c}
i+j \\
j
\end{array}\right) B\left(\gamma_{1}+i+1, \gamma_{2}+j\right) .
\end{aligned}
$$

Let $x_{1}, x_{2}, \ldots, x_{n}$ and $y_{1}, y_{2}, \ldots, y_{m}$ be independent observations from $X \sim \operatorname{EGEG}\left(p_{1}, \beta, \alpha, \gamma_{1}\right)$ and $Y \sim$ $\operatorname{EGEG}\left(p_{2}, \beta, \alpha, \gamma_{2}\right)$, respectively. The total log-likelihood function $\ell_{R}\left(\boldsymbol{\theta}^{*}\right)$, where $\boldsymbol{\theta}^{*}=\left(p_{1}, p_{2}, \beta, \alpha, \gamma_{1}, \gamma_{2}\right)^{T}$, reduces to

$$
\begin{aligned}
\ell_{R}\left(\boldsymbol{\theta}^{*}\right) & =n \log \gamma_{1}+m \log \gamma_{2}+(n+m) \log \left(\alpha \beta^{\alpha}\right) \\
& +n \log \left(1-p_{1}\right)+m \log \left(1-p_{2}\right) \\
& +(\alpha-1)\left(\sum_{i=1}^{n} \log x_{i}+\sum_{j=1}^{m} \log y_{j}\right) \\
& -\sum_{i=1}^{n}\left(\beta x_{i}\right)^{\alpha}-\sum_{j=1}^{m}\left(\beta y_{j}\right)^{\alpha} \\
& +\left(\gamma_{1}-1\right) \sum_{i=1}^{n} \log \left(1-e^{-\left(\beta x_{i}\right)^{\alpha}}\right) \\
& +\left(\gamma_{2}-1\right) \sum_{j=1}^{m} \log \left(1-e^{-\left(\beta y_{j}\right)^{\alpha}}\right) \\
& -\left(\gamma_{1}+1\right) \sum_{i=1}^{n} \log \left(1-p_{1} e^{-\left(\beta x_{i}\right)^{\alpha}}\right) \\
& -\left(\gamma_{2}+1\right) \sum_{j=1}^{m} \log \left(1-p_{2} e^{-\left(\beta y_{j}\right)^{\alpha}}\right) .
\end{aligned}
$$

Solving the system of nonlinear equation $U_{R}\left(\boldsymbol{\theta}^{*}\right)=$ $\left(\frac{\partial \ell_{R}}{\partial \hat{p_{1}}}, \frac{\partial \ell_{R}}{\partial \hat{p_{2}}}, \frac{\partial \ell_{R}}{\partial \hat{\beta}}, \frac{\partial \ell_{R}}{\partial \hat{\alpha}}, \frac{\partial \ell_{R}}{\partial \hat{\gamma_{1}}}, \frac{\partial \ell_{R}}{\partial \hat{\gamma_{2}}}\right)^{T}=\mathbf{0}$ numerically, the MLE of $\boldsymbol{\theta}^{*}$, say $\hat{\boldsymbol{\theta}^{*}}$, is obtained. The parameter $R$ can be estimated in this case with inserting $\hat{\boldsymbol{\theta}}^{*}$ in Equation (25).

\section{APPLICATION}

In this section, we use a real data set to illustrate an application of the EGEG distribution in data modeling. The following skewed data are given by Smith and Naylor [25] 


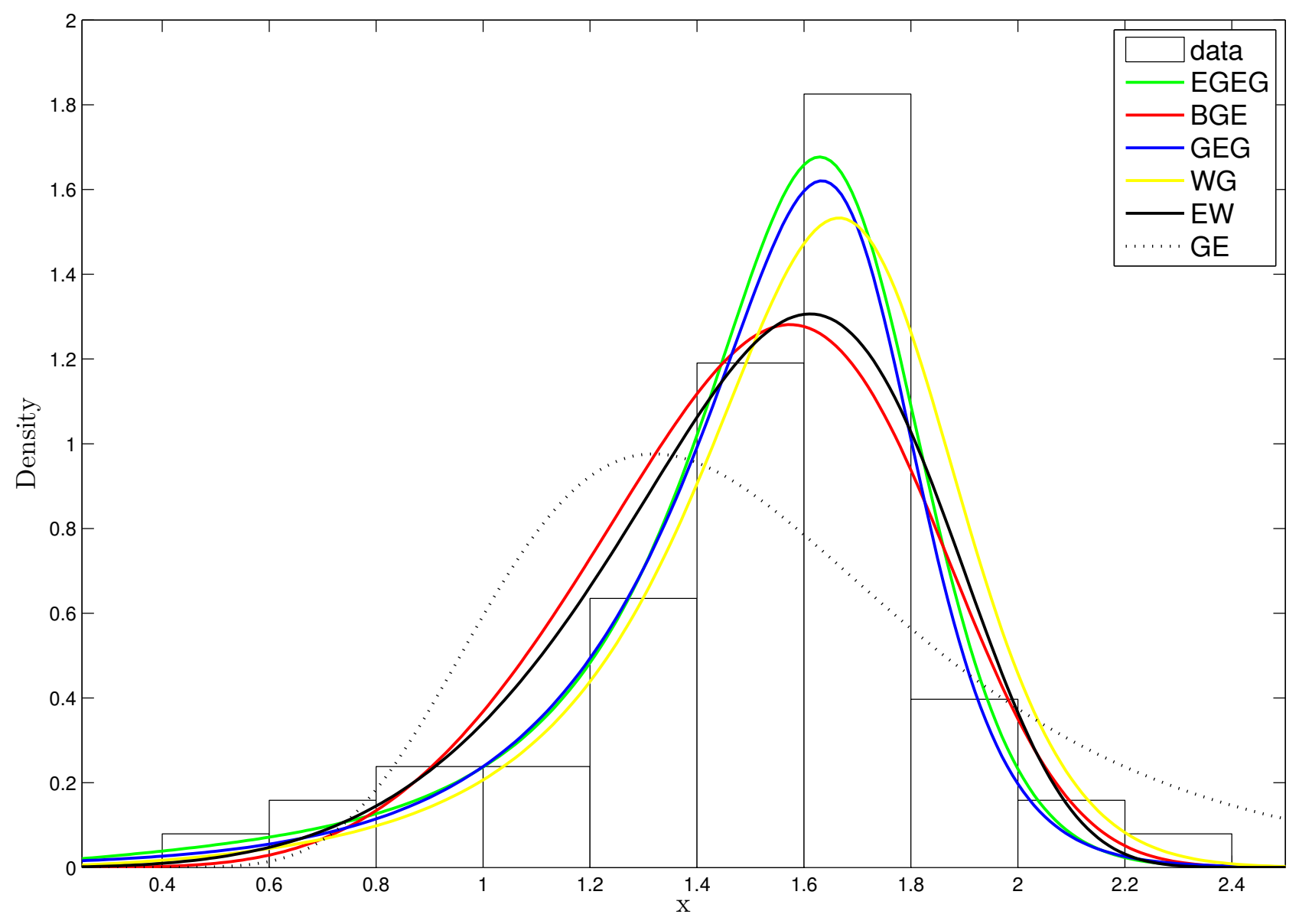

Fig. 3. Densities plots of the fitted models

and discussed further by Barreto-Souza et al. [26] which represent the strengths of $1.5 \mathrm{~cm}$ glass fibres, measured at the National Physical Laboratory, England. The data are:

$0.55,0.93,1.25,1.36,1.49,1.52,1.58,1.61,1.64,1.68,1.73$, $1.81,2.00,0.74,1.04,1.27,1.39,1.49,1.53,1.59,1.61,1.66$, $1.68,1.76,1.82,2.01,0.77,1.11,1.28,1.42,1.50,1.54,1.60$, $1.62,1.66,1.69,1.76,1.84,2.24,0.81,1.13,1.29,1.48,1.50$, $1.55,1.61,1.62,1.66,1.70,1.77,1.84,0.84,1.24,1.30,1.48$, $1.51,1.55,1.61,1.63,1.67,1.70,1.78,1.89$.

We fit the data to the $\operatorname{EGEG}(p, \beta, \alpha, \gamma)$ model and some sub-models such as the $W G(p, \beta, \alpha)$ model given in (12), $E W(\beta, \alpha, \gamma)$ model given in (15), $G E G(p, \beta, \gamma)$ model given in (14), and $G E(\beta, \gamma)$ model given in (15) when $\alpha=1$. As a competitive model, we fit the four-parameter beta generalized exponential (BGE) distribution of Barreto-Souza et al. [26] with pdf

$$
\begin{aligned}
& f(x ; a, b, \lambda, \alpha)=\frac{\alpha \lambda}{B(a, b)} e^{-\lambda x}\left(1-e^{-\lambda x}\right)^{\alpha a-1} \\
& \times\left\{1-\left(1-e^{-\lambda x}\right)^{\alpha}\right\}^{b-1} ; \quad x>0
\end{aligned}
$$

for $a>0, b>0, \lambda>0$, and $\alpha>0$ to these data. It is worth mentioning here that cdf (1) is still a cumulative dis-

Tab. 1. MLE, maximized log-likelihood, AIC, and BIC of the fitted models

\begin{tabular}{lcccc}
\hline Model & Parameters estimation & $\ell(\hat{\boldsymbol{\theta}})$ & AIC & BIC \\
\hline EGEG & $\hat{p}=-50.1217, \hat{\beta}=0.9628, \hat{\alpha}=3.0691, \hat{\gamma}=0.7314$ & -11.5146 & 31.0292 & 39.6017 \\
BGE & $\hat{a}=0.4125, \hat{b}=93.4655, \hat{\lambda}=0.92271, \hat{\alpha}=22.6124$ & -15.5995 & 39.1989 & 47.7715 \\
WG & $\hat{p}=-14.8952, \hat{\beta}=0.8594, \hat{\alpha}=3.1952$ & -13.6131 & 33.2262 & 35.5124 \\
EW & $\hat{\beta}=0.5820, \hat{\alpha}=7.2846, \hat{\gamma}=0.6712$ & -14.6755 & 35.3511 & 41.7805 \\
GEG & $\hat{p}=-111.2561, \hat{\beta}=0.61421, \hat{\gamma}=5.7807$ & -15.2068 & 34.4137 & 38.7000 \\
GE & $\hat{\beta}=2.6105, \hat{\gamma}=31.3032$ & -31.3835 & 66.7670 & 71.0532 \\
\hline
\end{tabular}


tribution function even for $p \leq 0$. Hence, we can consider the EGEG distribution defined by cdf (1) for any $p<1$ and also for the WG distribution as a sub-model. Therefore, our expressions for the moments, mgf, and order statistics are also valid for $-1 \leq p<1$. For $p<-1$, numerical computation should be investigated. (For a similar explanation in this connection, see [1]). To find the MLEs of the $E W(\beta, \alpha, \gamma)$ model, the initial values are the MLEs of the $G E(\beta, \gamma)$ model and $\alpha=1$. Subsequently, the initial values for finding the MLEs of the $E G E G(p, \beta, \alpha, \gamma)$ model can be MLEs of the $E W(\beta, \alpha, \gamma)$ model and a value near zero for $p$. As mentioned above, we see from Tab. 1 that the estimation of $p$ is not range-preserving.

Table 1 contains MLEs of the parameters, maximized $\log$-likelihood function $(\ell(\hat{\boldsymbol{\theta}}))$, Akaike information criterion (AIC), and Bayesian information criterion (BIC) as known criterions for comparing the fitted models.

According to AIC and BIC values in Table 1 and also Figures 3 and 4, it seems that the EGEG distribution gives a better fit than the BGE, EW, WG, GEG, and GE models. We have also performed Kolmogorov-Smirnov tests whose values (p-values) for the EGEG, BGE, WG, EW, GEG, and GE are 0.0925 (0.6209), 0.1673 (0.0520), 0.1575 (0.0786), 0.1461 (0.1226), 0.1522 (0.0969), and 0.2290 (0.0022), respectively. These results also confirm a good fit for the EGEG model.
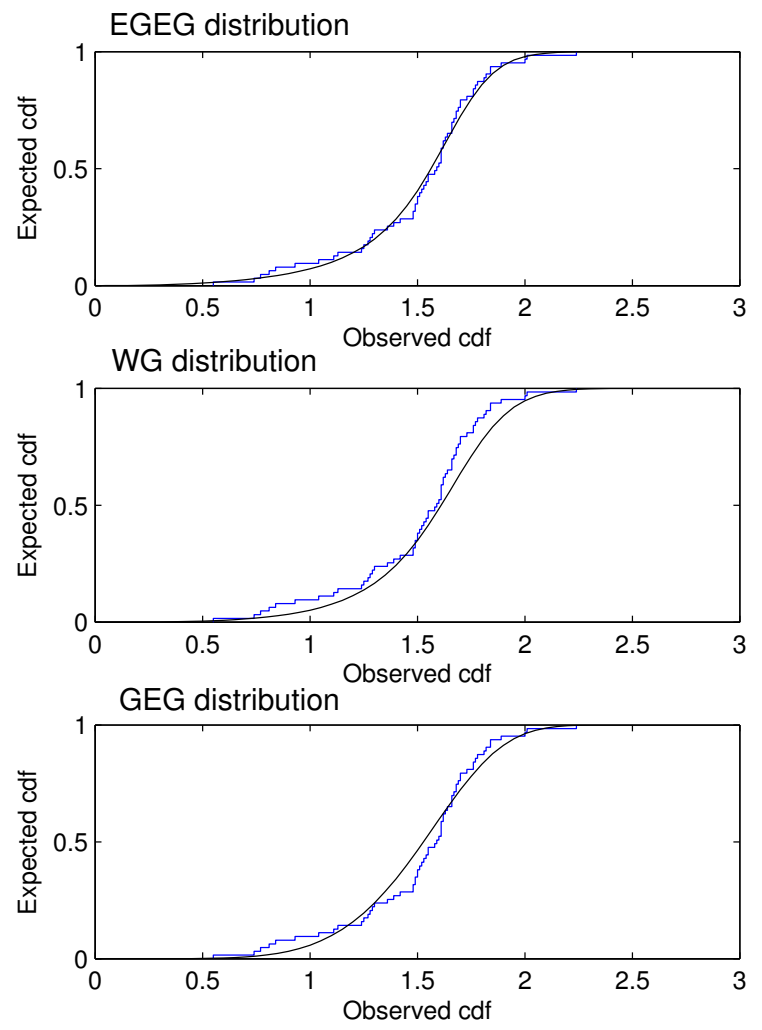

The asymptotic variance-covariance matrix of the MLEs for the EGEG model parameters, which is the inverse of the observed Fisher information matrix, is given by

$\boldsymbol{I}_{n}(\hat{\boldsymbol{\theta}})^{-1}=10^{3}$

$$
\times\left[\begin{array}{rrrr}
0.000151 & -0.000040 & 0.013441 & 0.000010 \\
-0.000040 & 0.000010 & -0.003910 & -0.000003 \\
0.013441 & -0.003913 & 0.000526 & 0.001954 \\
0.000010 & -0.000003 & 0.001954 & 0.000005
\end{array}\right]
$$

Thus, 95\% confidence intervals for the parameters are given by $p \in(-50.1217 \mp 0.7616), \beta \in(0.9628 \mp 0.1960)$, $\alpha \in(3.0691 \mp 1.4215)$, and $\beta \in(0.7314 \mp 0.1386)$.

In testing the null hypothesis $H_{0}$ : GEG versus the alternative hypothesis $H_{1}$ : EGEG, or equivalently, $H_{0}: \alpha=1$ versus $H_{1}: \alpha \neq 1$, the likelihood ratio (LR) test statistic value is 7.5068 (p-value $=0.0061)$ which is significant at $1 \%$ level. In addition, in testing the null hypothesis $H_{0}$ : WG versus the alternative hypothesis $H_{1}$ : EGEG, or equivalently, $H_{0}: \gamma=1$ versus $H_{1}: \gamma \neq 1$, the LR test statistic value is 4.1970 (p-value $=0.0405$ ) which is significant at $5 \%$ level.

\section{CONCLUDING REMARKS}

A new four-parameter lifetime distribution called the extended generalized exponential geometric (EGEG) dis-
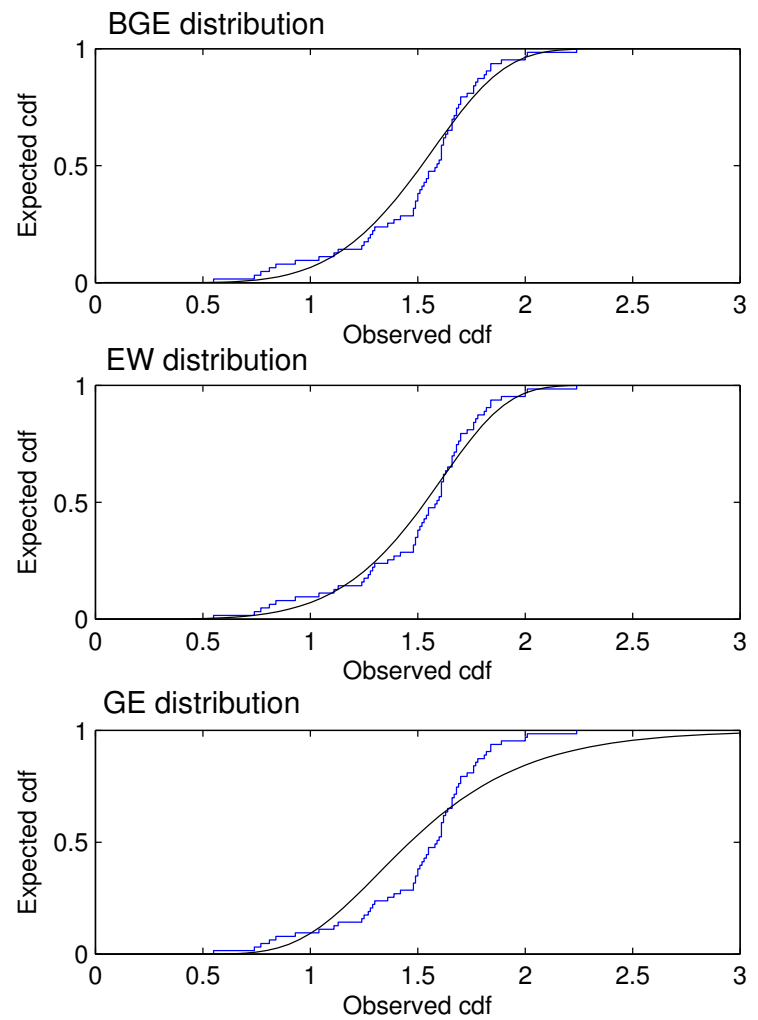

Fig. 4. Empirical cdf plots of the fitted models 
tribution is proposed by adding a resilience parameter to the Weibull-geometric (WG) distribution of Barreto-Souza et al. [2]. The new distribution also extends the generalized exponential geometric (GEG) distribution of Silva et al. [1] and includes some previously well-known distributions, such as the exponentiated Weibull (EW), Weibull, generalized exponential (GE), and the exponential-geometric (EG) distribution of Adamidis and Loukas [13] as sub-models. A characteristic of the EGEG distribution is that its failure rate function can be decreasing, increasing, bathtub, and upside-down bathtub depending on its parameters. We studied the basic distributional properties of the new distribution. Two general forms are given for the order statistics densities and stress-strength parameter. The maximum likelihood method is used to estimate the model parameters. We fitted the EGEG model to a real data set and found that the EGEG distribution yields a more satisfactory fit to the data than its rival models.

\section{Acknowledgments}

The author is grateful to the anonymous referee for helpful suggestions.

\section{Appendix}

Here, we intend to support the validity of inequality (11), used in the proof of Theorem II.2. For this purpose, let $0<t=e^{-(\beta x)^{\alpha}}<1$, for all $x>0, \alpha>0$, and $\beta>0$. Then, (11) reduces to

$$
\begin{aligned}
& \alpha \log t\left[(\gamma-1)(1-p t)^{2}-(\gamma+1) p(1-t)^{2}\right]< \\
< & (\alpha-1)(1-t)(1-p t) \\
\times & {[(\gamma+1) p(1-t)-(\gamma-1)(1-p t)] . }
\end{aligned}
$$

To prove (26), we make use of the following three Lemmas.

Lemma A.1 If $\gamma>1$ and $p \leq \frac{\gamma-1}{\gamma+1}$, then $(\gamma-1)(1-p t)^{2}-$ $(\gamma+1) p(1-t)^{2}>0$.

Proof. From the inequality $(1-p t)^{2}>(1-t)^{2}$ for all $t, p \in(0,1)$ and $p \leq \frac{\gamma-1}{\gamma+1}$, the assertion follows.

Lemma A.2 If $\gamma>1$ and $p \leq \frac{\gamma-1}{\gamma+1}$, then $\log t[(\gamma-1)(1-$ $\left.p t)^{2}-(\gamma+1) p(1-t)^{2}\right] \leq(t-1)\left[(\gamma-1)(1-p t)^{2}-(\gamma+\right.$ 1) $\left.p(1-t)^{2}\right]$.

Proof. Using the simple inequality $\log t \leq t-1$ for all $t>0$ and Lemma A.1, we have the proof.

Lemma A.3 If $\gamma>1, p \leq \frac{\gamma-1}{\gamma+1}$, and $\alpha>0$, then $\alpha(t-1)\left[(\gamma-1)(1-p t)^{2}-(\gamma+1) p(1-t)^{2}\right]<$ $(\alpha-1)(1-t)(1-p t)[(\gamma+1) p(1-t)-(\gamma-1)(1-p t)]$.
Proof. It is sufficient to prove that

$$
\begin{gathered}
\alpha\left[(\gamma+1) p(1-t)^{2}-(\gamma-1)(1-p t)^{2}\right] \\
-(\alpha-1)(1-p t)[(\gamma+1) p(1-t)-(\gamma-1)(1-p t)]<0 .
\end{gathered}
$$

For this, we consider the L.H.S of (27) as a function of $\alpha$. Thus, the L.H.S of (27) reduces to

$$
h(\alpha)=-[(\gamma+1) p(1-p) t(1-t)] \alpha+c(t),
$$

where $c(t)=(1-p t)[(\gamma+1) p(1-t)-(\gamma-1)(1-p t)]$ is a negative function, if $p \leq \frac{\gamma-1}{\gamma+1}$. It is clear that $h(\alpha)$ is a linear and decreasing function of $\alpha$. Further, $\lim _{\alpha \rightarrow 0} h(\alpha)=c(t)<0$ and, thus, (27) is proved.

Therefore, using Lemmas A.2 and A.3, the proof of (26) is evident.

\section{References}

[1] R.B. Silva, W. Barreto-Souza, and G.M. Cordeiro, A new distribution with decreasing, increasing, and upside-down bathtub failure rate, Comput. Statist. Data Anal. 54, 935-944 (2010).

[2] W. Barreto-Souza, A.L. de Morais, and G.M. Cordeiro, The Weibull-geometric distribution, J. Stat. Comput. Simul. 81, 645-657 (2011).

[3] A.W. Marshall and I. Olkin, Life distributions: Structure of nonparametric, semiparametric, and parametric families, Springer Science+Business Media, LLC, New York, 2007.

[4] G.S. Mudholkar and D.K. Srivastava, Exponentiated Weibull family for analyzing bathtub failure -rate data, Trans. Reliab. 42, 299-302 (1993).

[5] G.S. Mudholkar, D.K. Srivastava, and M. Freimer, The exponentiated Weibull family: A reanalysis of the bus-motor-failure data, Technometrics 37, 436-445 (1995).

[6] G.S. Mudholkar and A.D. Hutson, The exponentiated Weibull family: Some properties and a flood data application, Comm. Statist. Theory Methods 25, 3059-3083 (1996).

[7] R.D. Gupta and D. Kundu, Generalized exponential distributions, Aust. N. Z. J. Stat. 41, 173-188 (1999).

[8] S. Nadarajah and S. Kotz, The exponentiated type distributions, Acta Appl. Math. 92, 97-111 (2006).

[9] W. Barreto-Souza and F. Cribari-Neto, A generalization of the exponential-Poisson distribution, Statist. Probab. Lett. 79, 2493-2500 (2009).

[10] J.M.F. Carrasco, E.M.M. Ortega, and G.M. Cordeiro, A generalized modified Weibull distribution for lifetime modeling, Comput. Statist. Data Anal. 53, 450-462 (2008).

[11] C. Kus, A new lifetime distribution, Comput. Statist. Data Anal. 51, 4497-4509 (2007).

[12] C.D. Lai, M. Xie, and D.N.P. Murthy, A modified Weibull distribution, Trans. Reliab. 52, 33-37 (2003).

[13] K. Adamidis and S. Loukas, A lifetime distribution with decreasing failure rate, Statist. Probab. Lett. 39, 35-42 (1998).

[14] H. Bidram, J. Behboodian, and M. Towhidi, A new generalized exponential geometric distribution, Comm. Statist. Theory Methods. 42, 528-542 (2013).

[15] A. W. Marshall and I. Olkin, A new method for adding a parameter to a family of distributions with application to the exponential and Weibull families, Biometrika 84, 641-652 (1997). 
[16] V.K. Rohatgi, Distribution of order statistics with random sample size. Comm. Statist. Theory Methods 16, 3739-3743 (1987).

[17] S. Dharmadhikary and K. Joag-dev, Unimodality, convexity, and applications, Academic Press, Boston, 1998.

[18] R.E. Glaser, R.E., Bathtub and related failure rate characterizations, J. Am. Stat. Assoc. 75, 667-672 (1980).

[19] G.M. Cordeiro, A.B. Simas, and B.D. Stosic, Closed form expressions for moments of the beta Weibull distribution. Annals of the Brazilian Academy of Sciences. 83, 357-373 (2011).

[20] F. Famoye, C. Lee, and O. Olumolade, The beta-Weibull distribution, J. Statist. Theory Appl. 4, 121-136 (2005).

[21] C. Lee, F. Famoye, and O. Olumolade, Beta-Weibull distribution: Some properties and applications to censored data, J. Mod. Appl. Statist. Methods 6, 173-186 (2007).
[22] H. Bidram, J. Behboodian, and M. Towhidi, The beta Weibullgeometric distribution, J. Statist. Comput. Simul. 83, 52-67 (2013).

[23] A. Erdelyi, W. Magnus, F. Oberhettinger, and F.G. Tricomi, Higher Transcendental Functions, McGraw-Hill, New York, 1953.

[24] T. S. Ferguson, A course in large sample theory, Chapman and Hall, London, 1996.

[25] R.L. Smith and J.C. Naylor, A comparison of maximum likelihood and Bayesian estimators for the three-parameter Weibull distribution, J. Appl. Stat. 36, 358-369 (1987).

[26] W. Barreto-Souza, A.H. S. Santos, and G.M. Cordeiro, The beta generalized exponential distribution, J. Statist. Comput. Simul. 80, 159-172 (2010).

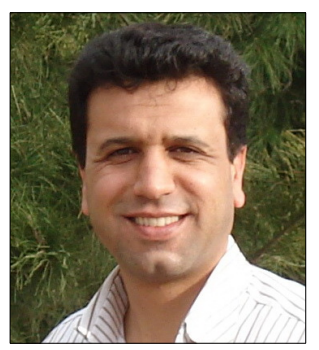

Hamid Bidram was born in Isfahan (Iran) in 1974. He received his MSc in Statistical Mathematics from Isfahan University of Technology (IUT-Iran) in 2000. He started his job as a lecturer at the University of Isfahan since 2000 and received his PhD degree in Distribution Theory from Shiraz University (Iran) in 2011. $\mathrm{He}$ is now an Assistant Professor of Statistics in Department of Statistics, Faculty of Sciences, University of Isfahan. He has the experience of being Head of Computer and Mathematics Faculty in Khansar Unit of University of Isfahan, during 2001 and 2002 and Head of Supervision and Evaluation Office in University of Isfahan from 2002 to 2006. His research interests include Statistical Computations, Distribution Theory, Data Modeling, Regression, and Applied Probability. In these fields he has written a book in Persian language and more than 15 scientific articles. 